\title{
REVISIÓN DE LA ESCALA DE ASERTIVIDAD DE RATHUS ADAPTADA POR LEÓN Y VARGAS (2009)
}

\author{
REVIEW OF THE RATHUS ASSERTIVENESS SCALE ADAPTED \\ BY LEON AND VARGAS (2009)
}

\author{
Marcela León Madrigal \\ marcela.leon@ucr.ac.cr
}

\begin{abstract}
Resumen
Se retomó el estudio desarrollado por León y Vargas (2009) para adaptar la escala Rathus a una muestra de estudiantes de educación superior costarricense. Se utilizaron los mismos datos con el fin de someter a prueba la estructura multidimensional del constructo, propuesta por dichos autores en el estudio original. También, se aporta nueva evidencia, basado en el análisis de estructuras de covarian$z a$, de la validez convergente y divergente de las puntuaciones de la escala. Se enfatiza la importancia práctica de distinguir los diferentes componentes de la conducta asertiva con el fin de diseñar intervenciones adecuadas.

Palabras claves: asertividad, conducta asertiva, análisis, convergente, divergente, Rathus
\end{abstract}

\section{Abstract}

This article revised a study develop by León \& Vargas (2009) to adapt Rathus scale to a sample of higher education costarican students. The same data was used in order to test the multidimensional structure of the construct proposed by these authors in the original study. Also, it provides new evidence based in the analysis of the structures of covariance, convergent and divergent validity in the scale scores. It emphasizes the practical importance of distinguishing the diferent components of the assertive behavior in order to design appropriate interventions.

Key words: Assertiveness, assertive behavior, convergent, divergent, analysis, Rathus.

1 Escuela de Psicología, Sede Rodrigo Facio, Universidad de Costa Rica. 


\section{Introducción}

El entrenamiento de la conducta asertiva ha sido un tema ampliamente estudiado desde los años setentas hasta la actualidad. Se ha reconocido su repercusión en las personas en diferentes ámbitos tales como la familia, el trabajo, la escuela y la salud física. Así mismo, se reconoce que personas con escasa conducta asertiva son proclives a enfrentar de manera inadecuada las demandas propias del medio social en que se desenvuelven (Rakos, 2006).

No existe una definición universalmente aceptada de conducta asertiva (León y Vargas, 2009). Tradicionalmente, se ha considerado que la asertividad se caracteriza por la emisión de conductas que expresan los derechos y sentimientos de la persona sin herir a los otros. También, algunos autores la han abordado como un continuo que va desde la pasividad, pasando por la asertividad, hasta la conducta agresiva (Caballo, 1993). Pese a esta falta de acuerdo, sí existe un relativo consenso acerca de los aspectos que caracterizan un comportamiento asertivo. Entre estos se pueden mencionar el reconocimiento de las deficiencias propias, dar y recibir cumplidos, iniciar y mantener interacciones con otras personas, expresar sentimientos positivos y negativos, externar expresiones no populares o diferentes, solicitar cambios de conductas de las otras personas y rechazar pedidos irracionales.

Como cualquier otro comportamiento, la asertividad está mediada por las cogniciones, racionales o irracionales, que forman el marco referencial de las personas. Así, personas con "diálogos internos negativos", entendidos como conducta encubierta cuyo contenido está cargado de verbalizaciones negativas sobre su desempeño o sobre sí mismos, tendrán más dificultades para emitir respuestas asertivas. También, debe considerarse el concepto de autoeficacia, ya que permite comprender la forma en la que las expectativas potencian o limitan el tipo de comportamiento emitido, de acuerdo con la evaluación que hacen las personas de sus capacidades. Por ejemplo, las personas no asertivas pueden tener expectativas negativas sobre el resultado de su comportamiento (Kail y Cavanaugh, 2011)
Más recientemente, se ha enfatizado que las conductas asertivas pueden tener diferentes consecuencias de acuerdo con el medio en el cual se emiten. Así, al asumir una postura asertiva la persona debe considerar la relación costo/beneficio en un contexto particular (Rakos, 2006).

El entrenamiento de la conducta asertiva es una herramienta fundamental en la práctica clínica, basta revisar la cantidad de trastornos en los que es mencionado el término asertividad en la Ecyclopedia of Cognitive Behavior Therapy editada por Freeman (2005), entre estos podemos citar algunos tales como: control de la ira, agresión y conducta antisocial en adolescentes, manejo de la ansiedad en personas tímidas, manejo del tiempo y pensamientos distorsionados, desarrollo de estrategias de comunicación para la contención y comprensión de pacientes con dolor crónico, pacientes con abuso de sustancias y presencia de enfermedades mentales, pacientes con retardo mental, juego patológico, manejo de la ira en ofensores sexuales y pedófilos, adquisición de estrategias para el control de depresión, ansiedad y culpa en personas que asumen el cuido de otras personas y desarrollo de la comunicación y manejo asertivo de cuidadores de pacientes terminales por ejemplo familiares, cuidadores y amigos, desarrollo de habilidades para la comunicación en pacientes que presentan Síntomas Médicos no Explicados tales como la fibromialgia, y la fatiga crónica.

Por otra parte, la práctica clínica psicológica se basa tanto en la experticia terapéutica como en el conocimiento científico. De hecho, el concepto de Psicoterapia Basado en la Evidencia (PBE) va más allá de la evidencia probabilista, entiéndase experimental o no, acerca de los métodos y técnicas de intervención. Rescata la experticia que el practicante requiere para la aplicación de los resultados relevantes de investigación, con el fin de desarrollar intervenciones con personas con necesidades y preferencias particulares (Huppert, Fabro \& Barlow, 2006). Se requiere dicha experticia debido a que es un proceso interpersonal complejo que se desarrolla bajo condiciones de incertidumbre y ambigüedad, así como bajo la presión de las necesidades urgentes del paciente (Goodheart, 2006). 
Por otra parte, una de las recomendaciones que ofrece Goodheart (2006) para mejorar el juicio clínico en la práctica cotidiana es el uso de pruebas psicológicas y métodos de evaluación conductual. En el marco de la terapia cognitivo conductual se ha apuntado la necesidad de abordar el concepto de asertividad en función de los aspectos situacionales y culturales en la que se emite la conducta, con el propósito de ofrecerle a la persona la forma más efectiva de influir en otros y resolver conflictos (Rakos, 2006). También, en el ámbito de la PBE el valor del contexto ha sido explícitamente reconocido; en esta dirección Comas-Diaz (2006) señala que la cultura media la psicoterapia.

La escala de asertividad de Rathus (RAS) ha sido uno de los instrumentos más utilizados en la evaluación de la conducta asertiva (Díaz, Ruiz \& Villalobos, 2012). Existe una adaptación para Costa Rica (RAS adaptada) realizada por León y Vargas (2009). Tomando como punto de partida los resultados de dicha investigación, así como la práctica clínica de la autora en la que ha aplicado de forma sistemática la RAS adaptada en el contexto del entrenamiento de la conducta asertiva, se propone revisar la hipótesis de la multidimensionalidad de dicha la escala. Se espera que esto se traduzca en una mejora en la evaluación y toma de decisiones de los practicantes, de manera que sea posible distinguir factores como el contexto, altamente relevantes desde el punto de vista de una evaluación clínica.

El presente estudio también busca verificar las evidencias de la validez encontradas por León y Vargas (2009) utilizando herramientas estadísticas más avanzadas, específicamente, el análisis de estructuras de covarianza.

\section{Metodología}

Se utilizaron los mismos datos del estudio original de León y Vargas (2009), los cuales fueron cedidos por el segundo autor. Con el objetivo de someter a prueba la hipótesis de la multidimensionalidad del constructo de asertividad evaluado por la RAS adaptada, se tomaron los resultados del análisis factorial exploratorio ortogonal en el que los citados autores encontraron seis subdimensiones. Estas fueron conceptualizadas tomando en cuenta los ítems de cada factor, la experiencia clínica de la autora y diversos aspectos relacionados con la conducta asertiva. 
Tabla 1

Escala de asertividad de Rathus (R.A.S adaptación León y Vargas, 2009)

\begin{tabular}{l} 
Subdimensión Items \\
\hline Demostrar disconformidad: capacidad para expresar molestia \\
o desagrado ante situaciones de carácter público en las que \\
existe la expectativa de satisfacer una necesidad planteada \\
expresamente por la persona y que espera que sea atendida \\
de una determinada manera.
\end{tabular}
de una determinada manera.

Items

3. Cuando la comida que me han servido en el restaurante no está hecha a mi gusto, me quejo con el mesero o mesera

17. En el restaurante o en cualquier sitio, protesto por un mal servicio

19. Si dos personas en el cine o en una conferencia están hablando demasiado alto, les pido que hagan silencio o se vayan a hablar a otra parte

20. Si alguien se cuela en una fila, le llamo abiertamente la atención

Manifestación de sentimientos y creencias: disposición para expresar de una forma clara, concisa y espontánea sentimientos, pensamientos y creencias que las personas pueden experimentar bajo ciertas circunstancias. También, comprende las respuestas motoras como la búsqueda y confrontación de personas involucradas.

5. Me esfuerzo, como la mayoría de la gente, por mantener mi posición

13. Soy abierto y franco en lo que respecta a mis sentimientos

14. Si alguien ha hablado mal de mí o me ha atribuido hechos falsos, lo (la) busco para saber qué es lo que pasa

21. Expreso mi opinión con facilidad

Eficacia: evaluación de la autoeficacia para manejar eventos. Es un juicio individual acerca de qué tan competente se puede ser en situaciones cotidianas de interacción social.

1. Pienso que mucha gente parece ser más agresiva y autoafirmativa que yo

2. He dudado en proponer o aceptar una cita con alguien por timidez

6. En realidad la gente se aprovecha de mí con frecuencia

7. Con frecuencia no sé qué decir a personas atractivas del otro sexo

16. Suelo reprimir mis emociones antes de hacer una escena

Interacción con organizaciones: capacidad para interactuar en el ámbito organizacional (institucional o empresarial) en situaciones en las cuales debe hacer solicitudes o responder a demandas específicas.

8. Evito llamar por teléfono a instituciones o empresas

9. En caso de solicitar un trabajo o la admisión en una institución, preferiría escribir cartas o enviar correos electrónicos, a realizar entrevistas personales

10. Me resulta embarazoso devolver un artículo comprado

Expresión de opiniones: temor a expresar de forma pública lo que se opina, por miedo a la burla o al juicio social negativo.

12. He evitado hacer preguntas por miedo a parecer tonto

18. Cuando me halagan con frecuencia, no sé qué responder

22. Hay veces en las que simplemente no puedo decir nada

Decir no: capacidad para expresar negación, comentarios opuestos a los intereses de los otros o la enunciación del no ante peticiones que se consideran inadecuadas o irracionales.

4. Cuando un vendedor se ha tomado el rato y la molestia de mostrarme un producto que después no quiero, paso mal rato al decir "NO"

11. Si un pariente cercano y respetado me molesta prefiero esconder mis sentimientos antes de expresar mi disgusto

15. Con frecuencia, paso un mal rato cuando tengo que decir que NO 
En la Tabla 1 se presentan las subdimensiones y los respectivos ítems de las escala, los cuales fueron evaluados por cada sujeto mediante una escala tipo Likert de 6 puntos.

Se seleccionó una muestra no probabilística con el fin de contar con estudiantes de diversas carreras y niveles educativos. La muestra estuvo constituida por 380 estudiantes del Centro Universitario de Cartago (C.U.C), a los cuales se les aplicó el formulario con las tres escalas (R.A.S., Asertividad / Sumisión y Obsesividad / Despreocupación). Además para establecer las normas de interpretación se realizaron adicionalmente 235 aplicaciones de la versión adaptada de la R.A.S. Por tanto, la muestra final de estandarización se formo por 615 casos provenientes de los tres lugares de aplicación Universidad de Costa Rica (U.C.R), Colegio Universitario de Cartago (C.U.C). y seminarios de actualización del Instituto Tecnológico de Costa Rica. (I.T.C.R.).

De los 615 casos 225 fueron hombres $36,6 \%$ y 390 mujeres $63,4 \%$. El nivel educativo se distribuyo de la siguiente manera: Primaria incompleta y completa 6 casos aproximadamente de un $1 \%$; Secundaria completa 358 casos con un 58,2\%; Técnico 77 casos con un 12,5\%; Bachillerato Universitario 78 casos con un $12.7 \%$; Licenciatura 65 casos con un 10,6\% y Maestría 27 casos con un $4,4 \%$.

En cuanto a las carreras, un $15,9 \%$ proviene de Psicología de la UCR; un $15.8 \%$ de Administración CUC; un 3,7\% de Electrónica CUC; 8,6 de Mecánica Dental CUC; un 4,1\% de Secretariado CUC; un 2,8 de Secretariado Inglés CUC; un 24,9 de Turismo CUC y un 22,3\% proviene de los Programa de actualización.

Con estos resultados se ejecutó un análisis factorial confirmatorio de segundo nivel, con el fin de confirmar la existencia de las dimensiones propuestas. Posteriormente, se utilizaron los datos de los 380 casos del estudio original a los que se les aplicó la escala RAS adaptada junto con las escalas de asertividad-sumisión y obsesividaddespreocupación del 21 subfactores de personalidad (21SFP) adaptado por Aguilar y González (1995). En el estudio original, mediante correlaciones bivariadas, se evaluó la convergencia y divergencia de los puntajes de las tres escalas con el fin de obtener evidencias de validez.
No es posible realizar el análisis usual de modelos multirasgo multimétodo con estructuras de covarianza debido a que solo se cuenta con un rasgo medido por dos métodos (ver Byrne, 2010 o Lévy \& Picón, 2006). Sin embargo, tomando en consideración que el argumento central de Camphlell y Fiske (1959) consiste en que cualquier medida de un constructo deberá mostrar correlaciones elevadas con otras medidas del mismo y débiles con medidas de diferentes constructos (Martínez, Hernández \& Hernández, 2006), se realizó un procesamiento alternativo adaptado a los datos existentes basado en análisis factoriales confirmatorios anidados.

En el estudio original, para evaluar la convergencia de los puntajes de la RAS adaptada se utilizó la escala de asertividad-sumisión del 21SFP. Esta define asertividad como la manifestación de la agresividad de una forma socialmente aceptada, la tendencia u orientación a mantenerse $o$ afirmarse en una posición de dominancia o ejercicio de la autoridad, en contraposición a asumir una actitud de subordinación o sumisión (Aguilar y González, 1995). Esta definición comparte algunos de los elementos con la RAS adaptada, pero no delimita el concepto de la misma forma, ya que enfatiza la posición y ejercicio de la autoridad. Para la divergencia, se usó la escala de obsesividad-despreocupación, rasgo que se define como la tendencia incontrolable e inoportuna de persistir o no en ciertas posiciones, ideas, pensamientos o actos (Aguilar y González, 1995).

Para realizar el análisis de convergencia y divergencia, se utilizaron parcelas de acuerdo a lo sugerido por Holt (2004). De esta forma se respetó la multitidimensionalidad del constructo Asertividad Rathus. En el caso de asertividad y obsesividad del 21SFP se combinaron aleatoriamente los ítems en 3 parcelas por factor. En todos los casos se calculó el puntaje medio.

Se estimaron cuatro modelos. El modelo uno no contiene restricciones en las covarianzas de los factores. En el modelo dos se restringen a cero las correlaciones entre las dos medidas de asertividad con la de obsesividad. Esto con el propósito de evaluar la convergencia. El modelo tres restringe a cero la correlación entre las mediciones de asertividad para evaluar la validez divergente. Con este mismo objetivo, el modelo cuatro 
fija en uno las correlaciones entre obsesividad y las dos medidas de asertividad. Siguiendo lo aconsejado por Byrne (2010), se evaluó el cambio absoluto en los índices $\chi^{2}, C F I$ y RMSEA de cada modelo con respeto al uno.

Los análisis factoriales se llevaron a cabo con el programa Analysis of Moment Structures (AMOS) versión 18. Los casos faltantes, con el fin de calcular los índices de modificación, se imputaron mediante el método de regresión.

\section{Resultados}

Se realizó un análisis factorial confirmatorio tomado las seis subdimensiones como factores endógenos latentes de primer orden y un único factor latente exógeno de segundo orden, en este caso Asertividad Rathus (ver figura 1). Con el fin de ajustar el modelo se permitió correlacionar errores basados en el contenido semántico del ítem siempre y cuando pertenecieran a la misma subdimensión. Se correlacionaron los siguientes errores: e3 y e17, e7 y e2, e22 y e18, e4 y e15. Estos corresponden a los ítems del mismo número (ver tabla 1). El índice normalizado de Mardia fue de 27.9, muy superior al máximo de 7 para que exista normalidad multivariante (Byrne, 2010).

El modelo obtuvo una $\chi^{2}$ de 452.14 con 199 grados de libertad. Se rechazó la hipótesis nula de un adecuado ajuste del modelo $(p<0.01)$, sin embargo, la literatura ha señalado ampliamente que esta prueba es muy sensible al tamaño de muestra (Hair, Black, Babin, \& Anderson, 2010). El GFI fue de 0.94 y el $A G F I$ de 0.92 , superior al mínimo de 0.9. Estos índices indican que la cantidad de varianza y covarianzas que explica el modelo es satisfactoria. Por otra parte, el $C F I$ fue de 0.91 y el RMSEA de 0.046 (con un PCLOSE de 0,91 ), ambos satisfactorios y por lo tanto indicando un adecuado ajuste de la solución de seis factores (Cea, 2002).

A nivel de los parámetros todas las cargas factoriales de primer nivel fueron estadísticamente significativas $(p<0.01)$ y mayores a 0.4. Solo en el caso del ítem 4 bajó a 0.37 . En cuanto a las cargas de segundo nivel, también fueron estadísticamente significativas $(p<0.01)$ y de una magnitud importante. Tal como se aprecia en la figura 1 , el factor de segundo nivel Asertividad Rathus explica cantidades importantes de la varianza de los factores de primer orden, específicamente, un $87 \%$ de Decir no y de expresión de opiniones; $84 \%$ de Eficacia; $47 \%$ de Interacción con organizaciones; $38 \%$ de Manifestación de sentimiento y opiniones y solo un $15 \%$ de Demostrar disconformidad. Estos resultados apoyan la hipótesis de la multidimensionalidad del constructo. Las cargas negativas

Figura 1

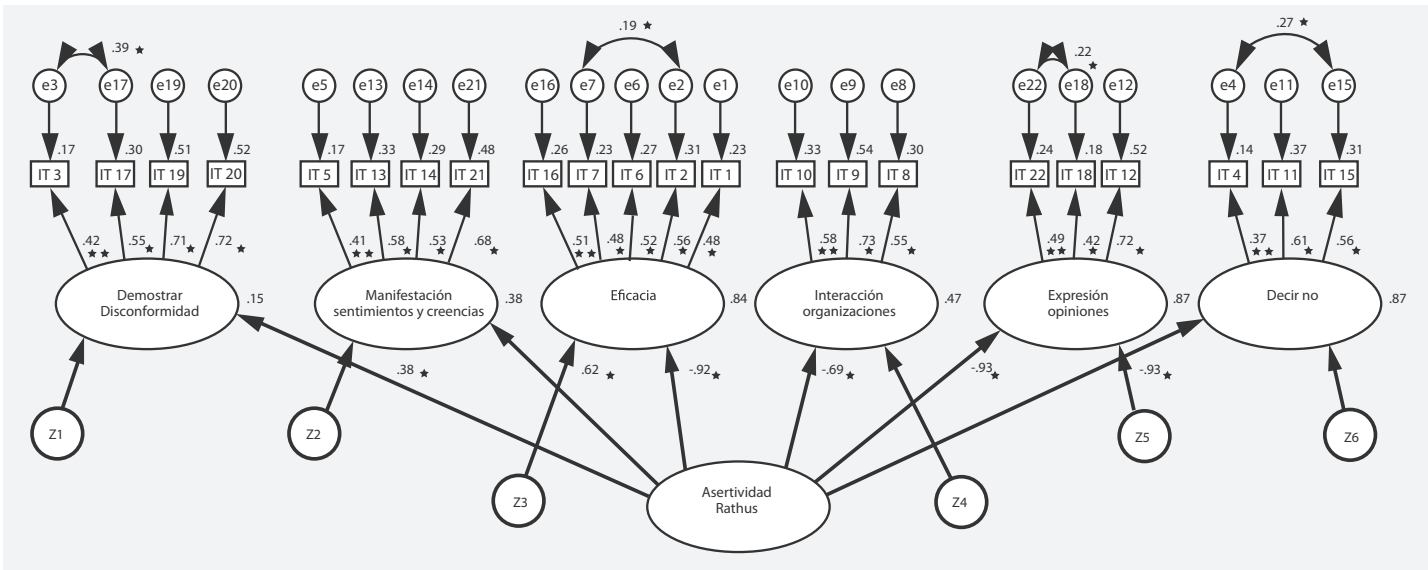

Análisis factorial confirmatorio de segundo nivel para la RAS adaptada. Coeficientes estandarizados estimados mediante el método de máxima versomilitud.

$* p<.01$.

** Fijado en uno para la estimación. 
se dan cuando los ítems de la subdimensión han sido redactados en sentido inverso de la escala del factor.

En cuanto a la convergencia y divergencia de los puntajes de las escalas como evidencias de validez, el modelo uno (ver figura 2) muestra los rasgos libremente correlacionados entre sí. Para ajustar el modelo se permitió la correlación entre los errores e5 y e6, correspondientes a las parcelas Manifestación de sentimientos y creencias y Demostrar disconformidad. Un examen de los ítems de cada una (ver tabla 1) evidencia la similitud semántica de ambas.
También, se permitió la correlación entre los errores e6 con e7 y e8 que pertenecen a la parcela 1 de asertividad del 21SFP. Igualmente, se correlacionó e5 con e8, correspondiente a la parcela 2 de este último constructo. Los ítems que componen estas parcelas fueron asignados aleatoriamente. Se muestran en la tabla 2 . Al respecto, resulta evidente la relación semántica de la mayoría de los ítems con las subdimensiones Manifestación de sentimientos y Demostrar disconformidad. No se permitieron todas las covarianzas entre los errores para no sobreajustar estadísticamente el modelo.

Tabla 2

Items que forman las parcelas del 21SFP

\begin{tabular}{ll}
\hline Parcela Items & \\
\hline \multicolumn{1}{c}{-Usted trata de seguir su propio camino indiferentemente a la oposición } \\
& -Usted puede hacer algo al respecto si alguien se mete delante de una fila, sin tocarle el turno \\
& -Usted expresa sus opiniones muy fuertemente \\
& -Usted cuando niño usualmente hacía lo que había dicho \\
& -Si alguien en el teatro tiene un sombrero puesto que le obstaculiza la visión, usted más bien \\
& podría buscar otro asiento, antes de pedirle que se lo quite \\
& -Usted usualmente, cuando está con un grupo de gente, es quien tomas las decisiones \\
& -Si usted ha recibido un mal servicio en un hotel o restaurante, prefiere dejarlo pasar, más \\
& bien que reclamar * \\
& -Usted prefiere permanecer en el fondo, más bien que irse adelante * \\
& -Usted piensa que competir deportivamente es más importante que ganar el evento * \\
& -Usted siempre debate un punto, si piensa que está en lo correcto *
\end{tabular}

*Items redactados en sentido inverso.

Una vez realizados los ajustes indicados se obtuvo una $\chi^{2}$ de 134.9 con 47 grados de libertad. El ajuste global del modelo es bueno con un CFI de 0.91 y un RMSEA de 0.07 tal como se aprecia en la tabla 3. Las correlación entre las dos medidas de asertividad es de 0.8 , estadísticamente significativa $(p<0.01)$. Las correlaciones de estas medidas con Obsesividad fueron bajas, menores a un valor absoluto de 0.24 y no significativas estadísticamente $(p<0.01)$.
Por otra parte, todas las cargas factoriales estimadas son mayores a 0.30 con excepción de Demostrar disconformidad, con 0.12; todas son estadísticamente significativas $(p<0.01)$. En este sentido, las magnitudes de las cargas y el ajuste general del modelo aportan evidencia de la convergencia y divergencia de los puntajes de las escalas y por tanto de la validez de las puntuaciones. 
Figura 2

Modelo uno de la secuencia de análisis factoriales confirmatorios

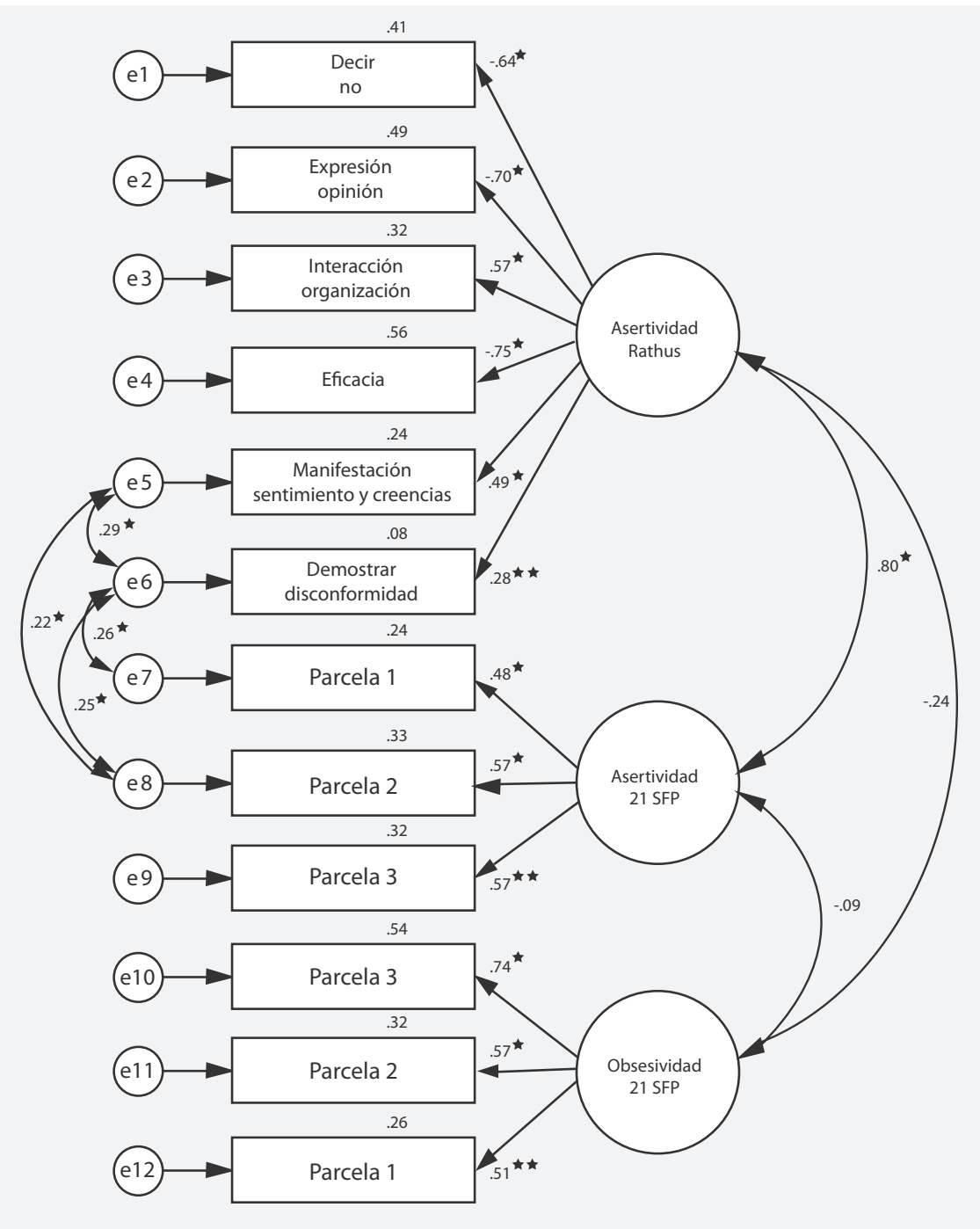

Modelo uno de la secuencia de análisis factoriales confirmatorios en el que los tres constructos correlacionan libremente. Coeficientes estandarizados estimados mediante el método de máxima versomilitud.

$* p<.01$.

** Fijado en uno para la estimación.

En el caso del modelo dos se fijaron en cero las covarianzas con Obsesividad. La correlación con este último constructo no es conceptualmente relevante, por lo que el ajuste de este modelo, en comparación con el uno, aporta evidencia de convergencia. En efecto, pese a esta restricción, el modelo continúa ajustando con un cambio en $\chi^{2}$ de 11.8 , apena superior a 10.6 , que corresponde a la variación mínima estadísticamente significativa con un alfa del $5 \%$. 
Figura 3

Modelo dos de la secuencia de análisis factoriales confirmatorios

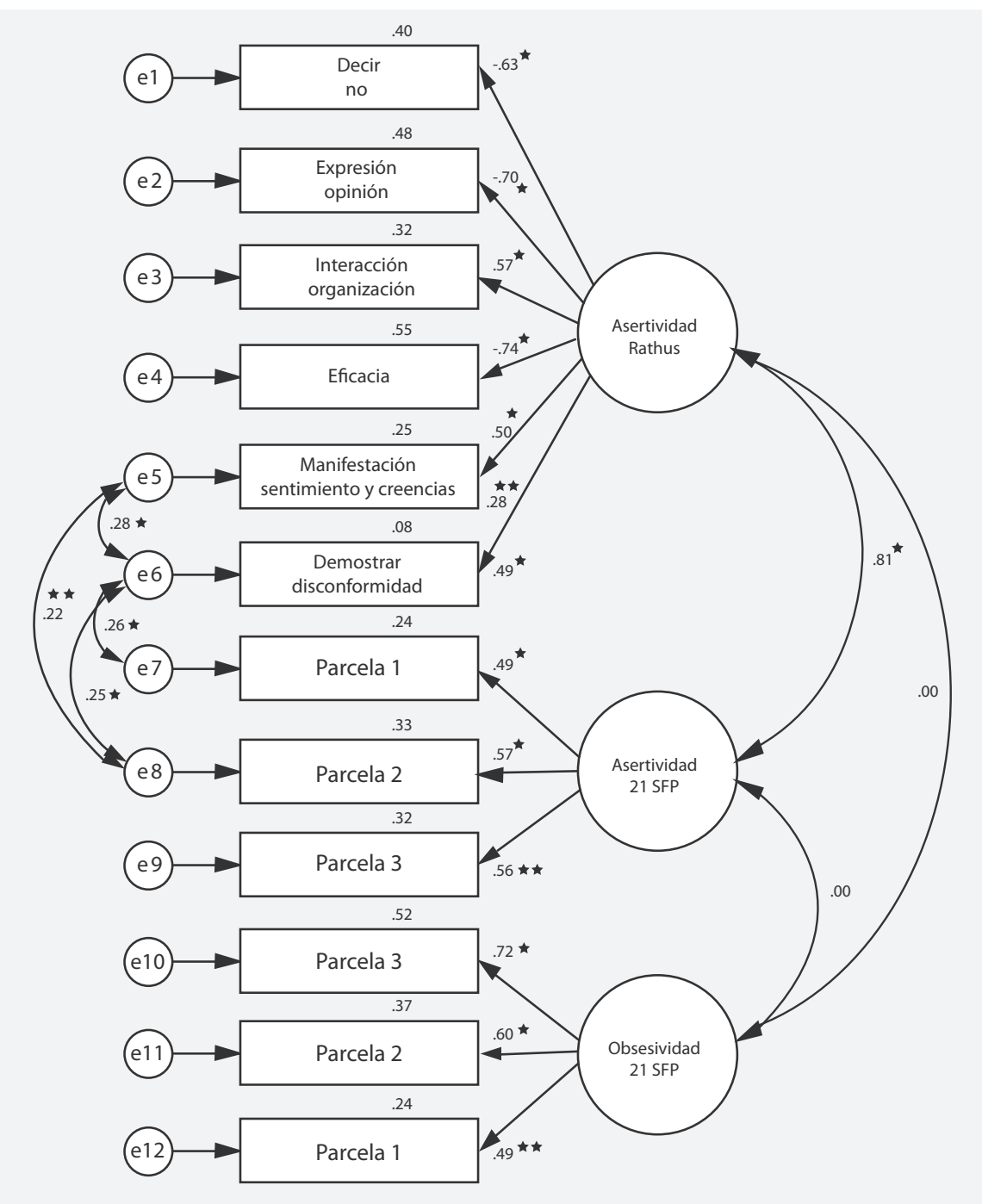

Modelo dos de la secuencia de análisis factoriales confirmatorios en el que las dos medidas de asertividad no se correlacionan con obsesividad. Coeficientes estandarizados estimados mediante el método de máxima versomilitud.

$* p<.01$.

** Fijado en uno para la estimación.

El CFI y el RMSEA se mantienen iguales, por lo que se concluye que el modelo ajusta. Las variaciones observadas en los modelos aportan evidencia de la convergencia, lo que a su vez apoya la validez de las puntuaciones del RAS adaptada en la muestra del estudio. 
Tabla 3

Índices de ajuste y cambio para la secuencia de modelos de análisis factoriales confirmatorios, $(n=380)$

\begin{tabular}{lccccccc}
\hline Modelo & $\chi^{2}$ & df & CFI & RMSEA & $\Delta \chi^{2}$ & $\Delta$ CFI & $\Delta R M S E A$ \\
\hline 1: los tres constructos correlacionan libremente & 134.9 & 47 & 0.91 & 0.07 & & & \\
$\begin{array}{l}\text { 2: las dos medidas de asertividad no se correlacionan } \\
\text { con obsesividad }\end{array}$ & 146.7 & 49 & 0.9 & 0.07 & $11.8^{*}$ & -0.01 & 0 \\
$\begin{array}{l}\text { 3: las dos medidas de asertividad no se correlacionan } \\
\begin{array}{l}\text { 4: las dos medidas de asertividad se correlacionan } \\
\text { perfectamente con obsesividad }\end{array}\end{array}$ & 255.73 & 48 & 0.79 & 0.11 & $120.8^{*}$ & -0.12 & 0.04 \\
\hline
\end{tabular}

$* p<0.01$

En el modelo tres se especificó en cero la correlación entre las dos medidas de asertividad. De esta forma, la diferencia en el ajuste respecto del modelo uno representa evidencia de divergencia. Como se observa en la tabla 3 se da un aumento de 120.8 en el $\chi^{2}$, cambio que es estadísticamente significativo y de una magnitud muy importante. Por otra parte, el $C F I$ disminuyó por debajo de un valor aceptable y el RMSEA aumentó por arriba de 0.08 , indicando un muy mal ajuste. Debido a que solo se permite la covariancia con obsesividad, entre mayor discrepancia con el modelo uno, mayor es la evidencia de divergencia que se obtiene. 
Figura 4

Modelo tres de la secuencia de análisis factoriales confirmatorios

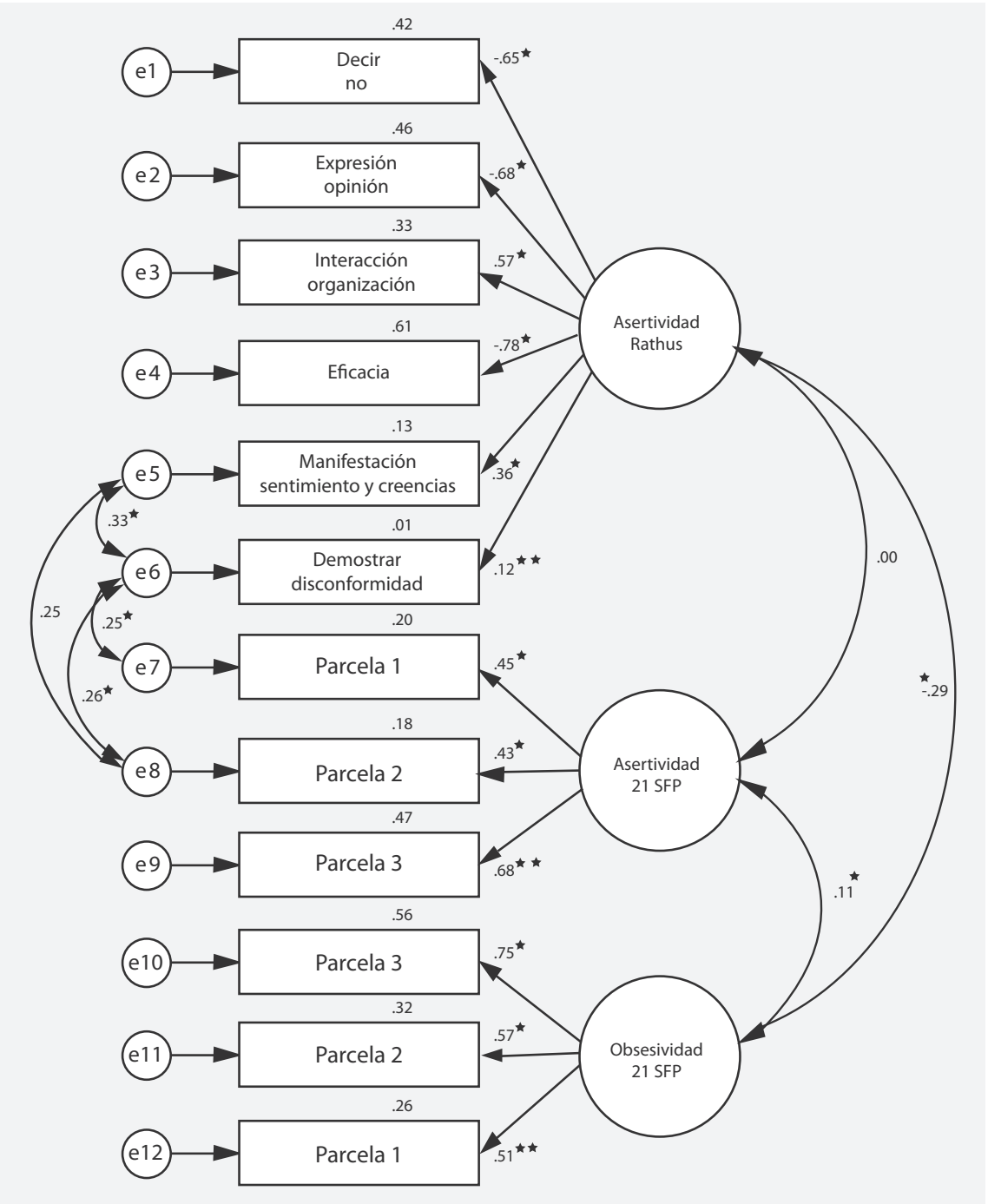

Modelo tres de la secuencia de análisis factoriales confirmatorios en el que las dos medidas de asertividad no se correlacionan. Coeficientes estandarizados estimados mediante el método de máxima versomilitud.

$* p<.01$.

** Fijado en uno para la estimación.

Para corroborar los resultados con respecto a la validez divergente, se estimó un cuarto modelo en el que se correlacionó perfectamente la medida de obsesividad con los dos constructos de asertividad (ver figura 5). En este caso, de forma similar al modelo tres, la magnitud de la discrepancia con respecto al modelo uno, evidencia la validez divergente. En efecto, tal y como se aprecia en la tabla 3 , es el modelo de peor ajuste. Obtuvo la mayor $\chi^{2}$ con 271.47 y 49 grados de libertad. El cambio fue de 136.8 con dos grados de libertad y fue estadísticamente significativo $(p<0.01)$. El CFI bajó a 0.78 y el RMSEA se mantuvo en 0.11 . 
Figura 5

Modelo cuatro de la secuencia de análisis factoriales confirmatorios

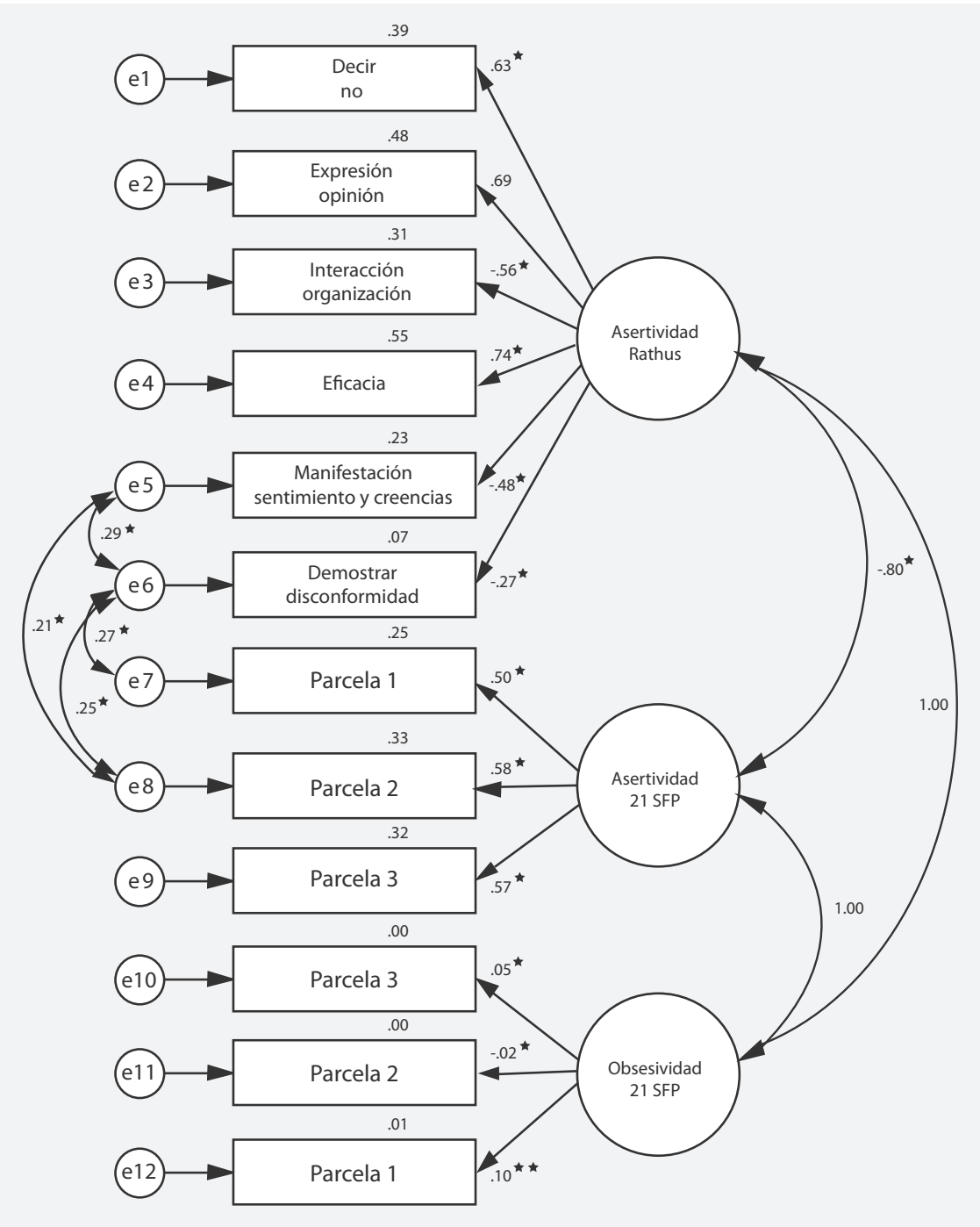

Modelo cuatro de la secuencia de análisis factoriales confirmatorios en el que las dos medidas de asertividad se correlacionan perfectamente con obsesividad. Coeficientes estandarizados estimados mediante el método de máxima versomilitud.

$* p<.01$.

$* * p<.05$.

** Fijado en uno para la estimación.

Se calculó el alpha de Cronbach para estimar la consistencia interna de cada una de las subdimensiones de la RAS adaptada. Este indicador se utilizó como estimación de la fiabilidad. Tal como han señalado Bearden, Netemeyer y Haws (2011) se han definido diferentes criterios con respecto al mínimo aceptable para este indicador, en un rango que va de 0.60 a 0.70 . Sin embargo, el número de ítems de la escala debe ser considerada, de manera que a mayor número el alpha tiende a aumentar (Field, 2009; Muñiz, 2002). 
Como se aprecia en la tabla 4, cinco de las seis subdimensiones obtuvieron alphas mayores a 0.6 , lo cual es aceptable lo cual tomando en consideración lo indicado por . Bearden, Netemeyer y Haws (2011) y el número reducido de ítems de cada sub escala (entre tres y cinco), se puede considerar aceptable. Solo el factor Decir no obtuvo 0.57. Pese a esto, es importante desarrollar más y mejores ítems para aumentar la fiabilidad de cada subescala.

Tabla 4

Alpha de cronbach para cada una de las subdimensiones de la RAS adaptada, $(\mathrm{n}=615)$

\begin{tabular}{lll}
\hline Subdimensión & Alpha de cronbach & Número de Items \\
\hline Demostrar disconformidad & 0.73 & 4 \\
Manifestación de sentimientos y creencias & 0.61 & 4 \\
Eficacia & 0.67 & 5 \\
Interacción con organizaciones & 0.62 & 3 \\
Expresión de opiniones & 0.62 & 3 \\
Decir no & 0.57 & 3 \\
\hline
\end{tabular}

Nota: el cálculo se ejecuto con las muestras de validación, $n=380$, y estandarización, $n=235$, para un total de 615 sujetos la de estala muestra incluye.

Finalmente, para efectos de contar con indicadores de comparación de los puntajes, a continuación se adjuntan las estadísticas descriptivas por subdimensión.

Tabla 5

Estadisticas descriptivas de las subdimensiones de la escala RAS adaptada, (n=615)

\begin{tabular}{lccccc}
\hline Subdimensiones & $\mathrm{M}$ & $\mathrm{DE}$ & Asimetría & Curtosis & Rango \\
\hline Demostrar disconformidad & 2.29 & 1.17 & 0.08 & -0.40 & 5.0 \\
Manifestación de sentimientos y creencias & 3.46 & 0.96 & -0.43 & -0.30 & 4.5 \\
Eficacia & 1.92 & 1.01 & 0.28 & -0.28 & 5.0 \\
Interacción con organizaciones & 3.43 & 1.15 & $-0,52$ & -0.30 & 5.0 \\
Expresión de opiniones & 2.33 & 1.19 & 0,04 & -0.50 & 5.0 \\
Decir no & 1.90 & 1.16 & 0,26 & 0.53 & 5.0 \\
\hline
\end{tabular}

Nota: el cálculo se ejecuto con las muestras de validación, $n=380$, y estandarización, $n=235$, para un total de 615 sujetos la de estala muestra incluye. 


\section{Conclusiones}

Se corroboró, mediante el análisis factorial confirmatorio, el hallazgo de León y Vargas (2009) de la estructura multidimensional del constructo de asertividad evaluado por la RAS adaptada en la muestra del estudio original. Esto en importante en la medida en la que lo que se considera o no asertivo está mediado por la cultura.

Cada una de las subdimenciones encontradas alude a aspectos importantes de la conducta asertiva que deben tomarse en cuenta en la práctica clínica, de acuerdo con el contexto cultural costarricense así como a las necesidades y expectativas del paciente, con respecto a la intervención.

Se corroboran las evidencias de convergencia y divergencia de los puntajes de la RAS adaptada, lo cual a su vez aporta validez a las inferencias que puedan realizarse a partir de las puntuaciones de la escala en la muestra del estudio.

Las confiabilidades moderadas encontradas indican que cada subdimensión debe trabajarse aún más, con el fin de añadir ítems y poder obtener una escala mucho más robusta.

Es necesario enfatizar que las medidas de posición y variabilidad presentadas en la tabla 5, solo pueden utilizarse como referencia, ya que no provienen de una muestra aleatoria. La construcción de baremos requiere de dicha aleatoriedad para calcular el error de estimación. Pese a esto, constituyen un punto de referencia, a partir del cual se puede triangular información con diferentes fuentes e instrumentos, con el fin de completar una adecuada evaluación clínica. En el contexto de la Terapia de la Conducta constituye un insumo para el análisis funcional.

Se le sugiere al practicante tomar las puntaciones de cada subdimensión como un indicador de un área que debe explorarse, de forma sistemática y a profundidad, con otras herramientas. Esto con el fin de identificar cuáles son los aspectos del entrenamiento en conducta asertiva que realmente benefician al paciente. No se recomienda (a partir de la literatura, la práctica clínica y estos hallazgos) utilizar de forma indiscriminada un determinado "paquete" de entrenamiento de conducta asertiva para todos los pacientes. Esto no solo constituye un error técnico y un mal uso de la Terapia de la Conducta, sino también una seria omisión ética.

\section{Referencias bibliográficas}

Aguilar, M. \& González, O.M. (1995). 21 Subfactores de la personalidad: Una nueva metodología de evaluación psicodiagnóstica. San José: Editorial de la Universidad de Costa Rica.

Bearden, W.,Netemeyer, R. \& Haws, K. (2011). Handbook of Marketing .Multi-item Measures for Marketing and Consumer Behavior Rearch.(Third Edition). Londres: SAGE Publications Ltd.

Byrne, B. (2010). Structural equation modeling with AMOS. Basics concepts, applications and programming. New York: Routledge.

Caballo, V.E. (1993) Manual de evaluación y entrenamiento de las habilidades sociales. Madrid: Siglo XXI de España editores.

Camphell, D. \& Fiske, D. (1959). Convergent and discriminat validation by multitraitMultimethod matrix. Applied Psychological Measureament, 15, 30-43.

Cea, M. (2002). Análisis multivariable. Teoría y práctica en la investigación social. Madrid: Editorial Síntesis.

Comas-Díaz, L. (2006). Cultural variation in the therapeutic relationship. En C. Goodheart, A. Kazdin, A. \& R. Sternberg. (Eds.), Evidence-based psychotherapy: Where practice and research meet, (pp. 81-105). Washington, DC: American Psychological Assn.

Díaz, M.I., Ruiz, M.A. \& Villalobos, A. (2012). El proceso en terapia de conducta: La evaluación conductual. En M.A. Ruiz, M.I. Díaz \& A. Villalobos. (Eds.), Manual de técnicas de intervención cognitivo conductual, (pp. 99-152). Bilbao: Desclée de Brouwer.

Goodheart, C (2006). Evidence, endeavor, and expertise in psychology practice. En C. Goodheart, A. Kazdin, A. \& R. Sternberg. (Eds.), Evidence-based psychotherapy: Where practice and research meet, 
(pp. 37-61). Washington, DC: American Psychological Assn.

Field, A. (2009).Exploratory Factor Analysis. En A. Field (Ed), Discovering statistics using SPSS.(Third Edition ed) (pp 619-680) Londres: SAGE Publications Ltd.

Freeman, A., (2005). Encyclopedia of Cognitive Behavior Therapy. New York: Springer.

Hair, J., Black, W., Babin, B. \& Anderson, R. (2010). Multivariate Data Analysis. USA: Prentice Hall.

Holt, J. (October, 2004). Item parceling in structural equations models for optimum solutions. Trabajo presentado en la 2004 Annual Meeting of the Mid-Western Educational Research Association, Columbus, $\mathrm{OH}$.

Huppert, J., Fabbro, A. \& Barlow, D. (2006). Evidence-based practice and psychological treatments. En C. Goodheart, A. Kazdin, A. \& R. Sternberg. (Eds.), Evidencebased psychotherapy: Where practice and research meet, (pp. 131-152). Washington, DC: American Psychological Assn.
Kail, R. \& Cavanaugh, J. (2011). Desarrollo humano: una perspectiva del ciclo vital. México: CENGAGE Learning.

León, M. \& Vargas, T. (2009). Validación y estandarización de la escala de asertividad de Rathus (R.A.S.) en una muestra de adultos costarricenses. Revista Costarricense de Psicología, 28 (41-42), 169-185.

Lévy, M. \& Picón, E. (2006). Los modelos multirasgos-multimétodos y la contrastación de la validez. En J. Lévy (Director), Modelización con estructuras de covarianza en Ciencias Sociales (pp. 373-402). España: netbiblo.

Martínez, M., Hernández, M. \& Hernández, M. (2006). Psicometría. Madrid: Alianza Editorial.

Muñiz, J. (2002). Teoría clásica de los test. Madrid: Ediciones Pirámide.

Rakos, R.F. (2006). Asserting and confronting. En O. Hargie (Edit). The Handbook of Communication Skills, (pp.345-381). New York: Routledge,Taylor and Francis Group. 
